\title{
Complete genome sequence of lovastatin producer Aspergillus terreus ATCC 20542 and evaluation of genomic diversity among $A$. terreus strains
}

\author{
Małgorzata Ryngajłło ${ }^{1}$ (D) Tomasz Boruta $^{2}$ (D) $\cdot$ Marcin Bizukojć $^{2}$ (D)
}

Received: 13 November 2020 / Revised: 30 December 2020 / Accepted: 20 January 2021 / Published online: 30 January 2021

(C) The Author(s) 2021

\begin{abstract}
In the present study, the complete genome of a filamentous fungus Aspergillus terreus ATCC 20542 was sequenced, assembled, and annotated. This strain is mainly recognized for being a model wild-type lovastatin producer and a parental strain of highyielding industrial mutants. It is also a microorganism with a rich repertoire of secondary metabolites that has been a subject of numerous bioprocess-related studies. In terms of continuity, the genomic sequence provided in this work is of the highest quality among all the publicly available genomes of $A$. terreus strains. The comparative analysis revealed considerable diversity with regard to the catalog of biosynthetic gene clusters found in A. terreus. Even though the cluster of lovastatin biosynthesis was found to be well-conserved at the species level, several unique genes putatively associated with metabolic functions were detected in A. terreus ATCC 20542 that were not detected in other investigated genomes. The analysis was conducted also in the context of the primary metabolic pathways (sugar catabolism, biomass degradation potential, organic acid production), where the visible differences in gene copy numbers were detected. However, the species-level genomic diversity of $A$. terreus was more evident for secondary metabolism than for the well-conserved primary metabolic pathways. The newly sequenced genome of A. terreus ATCC 20542 was found to harbor several unique sequences, which can be regarded as interesting subjects for future experimental efforts on $A$. terreus metabolism and fungal biosynthetic capabilities.
\end{abstract}

\section{Key points}

- The high-quality genome of Aspergillus terreus ATCC 20542 has been assembled and annotated.

- Comparative analysis with other sequenced Aspergillus terreus strains has revealed considerable diversity in biosynthetic gene repertoire, especially related to secondary metabolism.

- The unique genomic features of A. terreus ATCC 20542 are discussed.

Keywords Aspergillus terreus · Genome · Comparative genomics · Secondary metabolites

\section{Introduction}

Aspergillus terreus is a species associated mainly with three distinct scientific and engineering areas, namely, the

Małgorzata Ryngajłło

malgorzata.ryngajllo@p.lodz.pl

1 Institute of Molecular and Industrial Biotechnology, Lodz University of Technology, B. Stefanowskiego 4/10, 90-924 Lodz, Poland

2 Faculty of Process and Environmental Engineering, Department of Bioprocess Engineering, Lodz University of Technology, ul. Wolczanska 213, 90-924 Lodz, Poland invasive fungal infections (Lass-Flörl 2018; Pastor and Guarro 2014; Steinbach et al. 2004), the microbial production of itaconic acid, an important building block used in chemical industry (Klement and Buchs 2013; Krull et al. 2017; Okabe et al. 2009), and the biosynthesis of lovastatin, a secondary metabolite applied as a cholesterollowering drug in hypercholesterolemia treatments (Alberts et al. 1980; Barrios-González and Miranda 2010; Endo 2010; Hasan et al. 2019; Mulder et al. 2015). This microorganism is also considered to be a rich source of biotechnologically relevant enzymes, e.g., cellulases, xylanases, and lipases (Ghanem et al. 2000; Sethi et al. 2016; Sohail et al. 2016). Being a fungus of dual (i.e., harmful or useful) nature, extensively investigated both in 
the clinical and industrial contexts, A. terreus continues to serve as a subject of numerous research efforts (Vyas 2011).

The first complete genome sequence of $A$. terreus, specifically of the clinical strain A. terreus NIH2624, was provided by the BROAD Institute in 2005 and encompassed 29.36 megabase pairs $(\mathrm{Mb})$. Even today, the genome of A. terreus NIH2624 remains a high-quality reference for genetic studies on A. terreus. It took 11 years for the next genome of this species (strain 45A, also referred to as KM017963) to be published (Savitha et al. 2016). Within the next 4 years, the genomes of strains w25, T3 Kankrej, TN-484 (Kanamasa et al. 2019), M6925 (Palanivel et al. 2020), and IFO6365 (Takahashi et al. 2020) were submitted to the NCBI database. Surprisingly, the genome of A. terreus ATCC 20542, a model lovastatin-producing microorganism deposited in the American Type Culture Collection, has never been released. Originally isolated from soil in Spain (Alberts et al. 1980), this wild-type strain was applied in numerous bioprocess-related studies focused on the optimization of lovastatin production (see the review of Mulder et al. 2015 and references therein). Importantly, it was used in the mutagenesis experiments as a parent of high-yielding industrial strains (Buckland et al. 1989; Hendrickson et al. 1999; Vinci et al. 1991) and employed in the pioneering research on the lovastatin biosynthetic gene cluster (Kennedy et al. 1999). Even though Microbia (the former name of Ironwood Pharmaceuticals, Inc, Boston, Massachusetts, USA), an industrial biotechnology company, did share the sequence data of $A$. terreus ATCC 20542 in relation with the transcriptomic and metabolomic experiments on lovastatin production (Askenazi et al. 2003), the genomic fragment that was made publicly available encompassed only $0.14 \mathrm{Mb}$ of sequence and was far from being considered a completely sequenced genome.

If one aims to perform any system-level studies focused on elucidating the mechanisms of lovastatin biosynthesis, A. terreus ATCC 20542 should be regarded as a model strain and a highly recommended research subject. Having access to a high-quality, complete genome sequence is an essential prerequisite for an in-depth analysis of this kind, as well as for the efforts aimed at designing effective lovastatin-producing cell factories. In the context of uncovering the fungal biosynthetic repertoire, it is worth mentioning that the previous study demonstrated the rich catalog of secondary metabolites produced by $A$. terreus ATCC 20542 in addition to its main product, lovastatin (Boruta and Bizukojc 2016). Some of these molecules, e.g., (+)-geodin, asterric acid, and butyrolactone I, display interesting biological activities, e.g., act as enzyme inhibitors or antibiotics, what makes them the potential candidates for future drug discovery efforts (Kitagawa et al. 1994; Ohashi et al. 1992; Rinderknecht et al. 1947; Sato et al. 2005; Shinohara et al. 2000; Takatsuki et al. 1969). As the capability to produce a certain set of secondary metabolites is known to be strain-specific, studying the biosynthetic gene clusters in A. terreus ATCC 20542 still requires a fully sequenced genome. In our previous study, the dataset corresponding to A. terreus NIH2624 was chosen to be applied for bioinformatic analyses of fungal gene clusters solely due to the fact that the genome of our true experimental subject, namely A. terreus ATCC 20542, was still unavailable (Boruta and Bizukojc 2014).

Six out of seven complete genomes of $A$. terreus previously deposited into the NCBI database were released between the years 2016 and 2020, what reflects the recently observed rapid build-up of sequence data related to this microorganism. Generally, as the number of sequenced genomes of fungi representing a particular species increases, the door opens for comparative genomic analysis at the species level. It should be emphasized that the strains of $A$. terreus previously subjected to sequencing were isolated from diverse environments and represented various characteristics. Until now, there has been no comprehensive study addressing the genomic differences and similarities within the rapidly expanding group of sequenced $A$. terreus strains.

The goals of the present study were to provide a highquality whole-genome assembly and annotation of $A$. terreus ATCC 20542 and to assess the diversity observed among the sequenced genomes of $A$. terreus strains.

\section{Materials and methods}

\section{Cultivation conditions}

A. terreus ATCC 20542 was purchased from the American Type Culture Collection (ATCC) and inoculated onto agar slants according to the recommendations specified by the ATCC. The spores obtained on the slants were then employed for the inoculation of liquid medium.

The submerged cultivations were carried out with the use of Certomat ${ }^{\circledR}$ BS-1 (B. Braun Biotech International, Melsungen, Germany) rotary shaker. The temperature was set at $28^{\circ} \mathrm{C}$, the rotary speed was kept at $110 \mathrm{~min}^{-1}$. The fungal biomass proliferated in flat-bottom flasks (working volume $150 \mathrm{ml}$, total volume $500 \mathrm{ml}$ ).

The first stage of the cultivation process (i.e., the preculture) was initiated by transferring the spores to liquid medium ( $10^{9}$ fungal spores per liter of medium) with the use of a sterile pipette. After $24 \mathrm{~h}$ of cultivation, the second stage of the process was initiated by inoculating $150 \mathrm{ml}$ of the production medium with $7 \mathrm{ml}$ of the preculture. The process was continued for $48 \mathrm{~h}$, and then, the biomass was separated from the liquid medium by filtration. The harvested biomass (fungal pellets) was washed thoroughly with PBS buffer (phosphate-buffered saline) and stored at $-80^{\circ} \mathrm{C}$. 
The medium composition was as follows: lactose, $20 \mathrm{~g} \mathrm{l}^{-1}$ (10 $\mathrm{g} \mathrm{l}^{-1}$ in preculture); yeast extract, $4 \mathrm{~g} \mathrm{l}^{-1}\left(8 \mathrm{~g} \mathrm{l}^{-1}\right.$ in preculture); $\mathrm{KH}_{2} \mathrm{PO}_{4}, 1.51 \mathrm{~g} \mathrm{l}^{-1} ; \mathrm{NaCl}, 0.4 \mathrm{~g} \mathrm{l}^{-1} ; \mathrm{MgSO}_{4}$. $7 \mathrm{H}_{2} \mathrm{O}, 0.51 \mathrm{~g} \mathrm{l}^{-1}$; biotin, $0.04 \mathrm{mg} \mathrm{l}^{-1}$; and $1 \mathrm{ml} \mathrm{l}^{-1}$ of trace element solution of the following composition: $\mathrm{ZnSO}_{4} \cdot 7 \mathrm{H}_{2} \mathrm{O}$, $1 \mathrm{gl}^{-1} ; \mathrm{Fe}\left(\mathrm{NO}_{3}\right)_{3} \cdot 9 \mathrm{H}_{2} \mathrm{O}, 2 \mathrm{~g} \mathrm{l}^{-1} ; \mathrm{MnSO}_{4}, 50 \mathrm{mg} \mathrm{l}^{-1} ; \mathrm{Na}_{2} \mathrm{~B}_{4} \mathrm{O}_{7}$. $10 \mathrm{H}_{2} \mathrm{O}, 100 \mathrm{mg} \mathrm{l}^{-1} ; \mathrm{Na}_{2} \mathrm{MoO}_{4} \cdot 2 \mathrm{H}_{2} \mathrm{O}, 50 \mathrm{mg} \mathrm{l}^{-1}$; and $\mathrm{CuSO}_{4}$. $5 \mathrm{H}_{2} \mathrm{O}, 250 \mathrm{mg} \mathrm{l}^{-1}$. The sterilization was performed in an autoclave for $30 \mathrm{~min}$ at $121^{\circ} \mathrm{C}$. The $\mathrm{pH}$ was set to 6.5 with the use of $\mathrm{NaOH}$ solution prior to sterilization.

\section{Isolation and sequencing of genomic DNA}

Genomic DNA of A. terreus ATCC 20542 was isolated using the SDS/Phenol method as described previously (Wilson 1987; Nowak et al. 2019). DNA quality control was performed by measuring the absorbance at 260/230, template concentration was determined using Qubit fluorimeter (Thermo Fisher Scientific, Waltham, USA), and DNA integrity was analyzed by $0.8 \%$ agarose gel electrophoresis and by PFGE using Biorad CHEF-III instrument (BioRad, Hercules, USA).

Paired-end sequencing library was constructed using the NEB Ultra II FS Preparation Kit (New England Biolabs, Beverly, USA) according to the manufacturer's instructions. Library was sequenced using an Illumina MiSeq platform (Illumina, San Diego, USA) with $2 \times 300$ paired-end reads. Sequence quality metrics were assessed using FASTQC (http://www.bioinformatics.babraham.ac.uk/projects/fastqc/; (Andrews 2010)).

The long reads were obtained using the MinION sequencer (Oxford Nanopore Technologies (ONT), Oxford, UK). Prior to long-read library preparation, genomic DNA was sheared into $20 \mathrm{~kb}$ fragments using Covaris g-Tube (Covaris, MA, USA) followed by size selection on Bluepippin instrument (Sage Science, Beverly, USA). DNA fragments above $10 \mathrm{~kb}$ were recovered using PAC30kb cassette. A total of $5 \mu \mathrm{g}$ of recovered DNA was taken for 1D library construction using a SQK-LSK109 kit (Oxford Nanopore Technologies (ONT), Oxford, UK) and $0.5 \mu \mathrm{g}$ of final library was loaded into a R9.4.1 flowcell (Oxford Nanopore Technologies (ONT), Oxford, UK) and sequenced on MinION sequencer (Oxford Nanopore Technologies (ONT), Oxford, UK).

\section{Genome assembling}

Raw nanopore data was basecalled using Guppy (v. 3.0; Oxford Nanopore Technologies, Oxford, UK). After quality filtering using NanoFilt (De Coster et al. 2018) and residual adapter removal using Porechop (https://github.com/rrwick/ Porechop), obtained dataset was quality checked using NanoPlot (De Coster et al. 2018). Long nanopore reads were assembled using Flye (v. 2.5; (Kolmogorov et al. 2019)).
Flye-assembled contigs were further polished using Illumina sequencing reads and Oxford Nanopore ont-assembly-polish pipeline (https://github.com/nanoporetech/ont-assemblypolish).

\section{Annotation and analysis of $A$. terreus ATCC 20542 and the published $A$. terreus genomes}

Genome assemblies of 8 A. terreus strains were downloaded from NCBI (access date: March 2020). Sequences of all analyzed A. terreus genomes were annotated using AUGUSTUS (v. 3.3.2; (Stanke and Waack 2003)) employing the supplied training annotation files for A. terreus. Ribosomal RNA (rRNA) genes were predicted in the A. terreus ATCC 20542 genome using barrnap (v. 0.9; Seemann T., https://github. com/tseemann/barrnap). Prediction of transfer RNA (tRNA) genes was done using the tRNAscan-SE (v. 2.0.5; (Chan and Lowe 2019)). Presence of signal peptide in the sequences of proteins was done using SignalP (v. 5.0; (Almagro Armenteros et al. 2019)). Transmembrane helices were predicted using Phobius server (access date: August 2020 (Käll et al. 2007)). Eukaryotic orthologous group (KOG) categories were assigned to the predicted A. terreus ATCC 20542 proteome by the WebMGA server (Wu et al. 2011). KEGG Automatic Annotation Server (KAAS; https://www.genome. $\mathrm{jp} /$ tools/kaas/) was used to perform KEGG orthology search employing the assignment method of 'bi-directional best hit' $(\mathrm{BBH})$ against Eurotiomycetes species. The proteomes of the analyzed species were functionally characterized using software package InterProScan (v. 5.19; (Jones et al. 2014)) with the option to scan for Pfam collection of protein families. Carbohydrate-active enzymes (CAZyme; Lombard et al. 2014) were annotated using dbCAN2 meta server (Zhang et al. 2018) with the option to use HAMMER search against dbCAN HMM database, DIAMOND search against CAZy database, and Hotpep search against the PPR short peptide library. Candidate proteins found by at least two methods were kept for further analysis. Secondary metabolite clusters were predicted using antiSMASH for fungi (v. 5.1.1; (Medema et al. 2011; Blin et al. 2019)). Orthologs were searched between the proteomes using Proteinortho program (version 6.0.14; (Lechner et al. 2011)). ANI analysis performed using PYANI (v. 0.2.9) python program employing BLAST+ program (Camacho et al. 2009; Pritchard et al. 2016). The UPGM tree based on ANI-1 values was calculated using phangorn R package (Schliep 2011).

\section{Genome sequence and annotation data availability}

The assembled genome, together with annotation, was deposited at the NCBI database under the BioProject: PRJNA622971. 


\section{Results}

\section{Sequence and general features of the genome of A. terreus ATCC 20542}

The sequencing and assembling of A. terreus ATCC 20542 genome produced 8 contigs and 1 scaffold of the total size of $30,360,760$ bp and the GC content of $52.2 \%$ (Table 1; Fig. 1a). The estimated number of protein-coding genes in the genome was equal to 10,505 . Forty-five rRNA genes were predicted. Out of 196 predicted tRNA genes, 18 were classified as pseudogenes which may not be functional. The signal peptide was detected in the sequence of 1025 proteins, whereas 2502 were predicted to contain transmembrane regions. Based on InterProscan search, Pfam domains were assigned to 8223 proteins ( $78 \%$ of the proteome). A total of 6533 of proteins were assigned to KOG categories representative of four main function types: information storage and processing, cellular processes and signaling, metabolism, and poorly characterized proteins (Fig. 1b).

\section{Phylogenetic analysis and comparative genomics of A. terreus strains}

The genomes of other seven A. terreus strains were downloaded from the NCBI database (Table 2). The average genome size of an A. terreus strain is $29.6 \mathrm{Mb}$ and the average $\mathrm{GC}$ content is $52 \%$. On average, the A. terreus genome encodes 10,421 genes (excluding the A. terreus T3 Kankrej genome due to high assembly fragmentation). Only the genome

Table 1 Assembly statistics and general features of A. terreus ATCC 20542 genome
Total number of reads-Illumina

Total number of reads-ONT

Genome size (bp)

Number of contigs/scaffolds

Scaffold N50 (bp)

Scaffold L50

$\% \mathrm{GC}$

Genome coverage-Illumina

Genome coverage-ONT

Number of protein coding genes

rRNA genes

tRNA genes

Proteins with signal peptide

Proteins with transmembrane helices

Proteins with predicted Pfam domain

Proteins assigned to KOG

Predicted CAZy proteins
$17,481,351$

392,432

$30,360,760$

9

$4,253,827$

3

52.2

$163 \times$

$140 \times$

10,505

45

196

1025

2502

8223

6,533

579 assembly of A. terreus NIH 2624 includes mitochondrial DNA sequence (1 scaffold of 32,827 bp with no predicted genes).

According to the results of the phylogenetic analysis based on the ANI values, three main clades can be noticed (Fig. 2), i.e., one which groups genomes of the A. terreus NIH 2624, A. terreus 45A, A. terreus M6925, A. terreus w25, and A. terreus T3 Kankrej strains; the second clade with two A. terreus ATCC 20542 genomes; the third and the most separated from the first two, clade grouping the genomes of IFO 6365 and TN-484 strains.

\section{Primary carbon metabolism and plant biomass degradation}

In order to gain insight into the conservation of the primary metabolism pathways in A. terreus strains, first their potential to utilize carbohydrates was compared by investigating the presence of orthologs of respective catabolic enzymes based on sequence similarity with proteins of Aspergillus niger ATCC 1015 (Table S1 of Online Resource 1). The investigated carbon sources included cellobiose, D-fructose, D-galactose, D-glucose, lactose, D-mannose, maltose, sucrose, and Dxylose. A similar analysis was conducted with regard to the genes involved in the production of organic acids, such as citric, fumaric, malic, succinic, and gluconic acid (Table S2 of Online Resource 1). In general, orthologs of all the investigated enzymes were found in every A. terreus genome and their copy number agreed, apart from only a few cases such as, e.g., glucose oxidase and D-xylulose reductase.

Next, to evaluate the plant biomass-degrading ability across A. terreus strains, the genes from CAZy (Carbohydrate-Active enZYmes) families were predicted (Table 3). The total number of CAZy genes predicted in a single genome varied from 450 to 483 depending on the strain, with the highest number found in the genome of $A$. terreus M6925. The differences in the numbers of genes involved in the degradation of cellulose, xylan, galactomannan, and xyloglucan did not exceed 1 among the tested genomes, whereas the abundance of sequences associated with pectin, inulin, and starch turned out to be somewhat more varied (Table 3). The analysis revealed that the genome of A. terreus M6925 was particularly enriched in inulinaseencoding genes. It was also noticed that the genomes of the two A. terreus strains of the second phylogenetic clade, i.e., A. terreus IFO 6365 and TN-484, contained the lowest number of genes required for the degradation of cellulose, pectin, and starch.

Even though the set of genes associated with primary metabolic pathways in A. terreus ATCC 20542 at first glance does not seem to be exceptional compared with other representatives of the species, a small set of unique sequences having no counterparts in other A. terreus strains has been found 
Fig. 1 Characteristics of A. terreus ATCC 20542 genome. a Map of A. terreus ATCC 20542 genome composed of 8 contigs and one scaffold color-coded according to GC content (red is more than $57 \%$, yellow less than $50 \%$, and the blued shades represent a linear gradient between the both numbers). b KOG categories in A. terreus ATCC 20542 proteome annotated by the WebMGA web server. Proteins assigned to more than one category were counted in each of them a

A. terreus ATCC 20542 genome

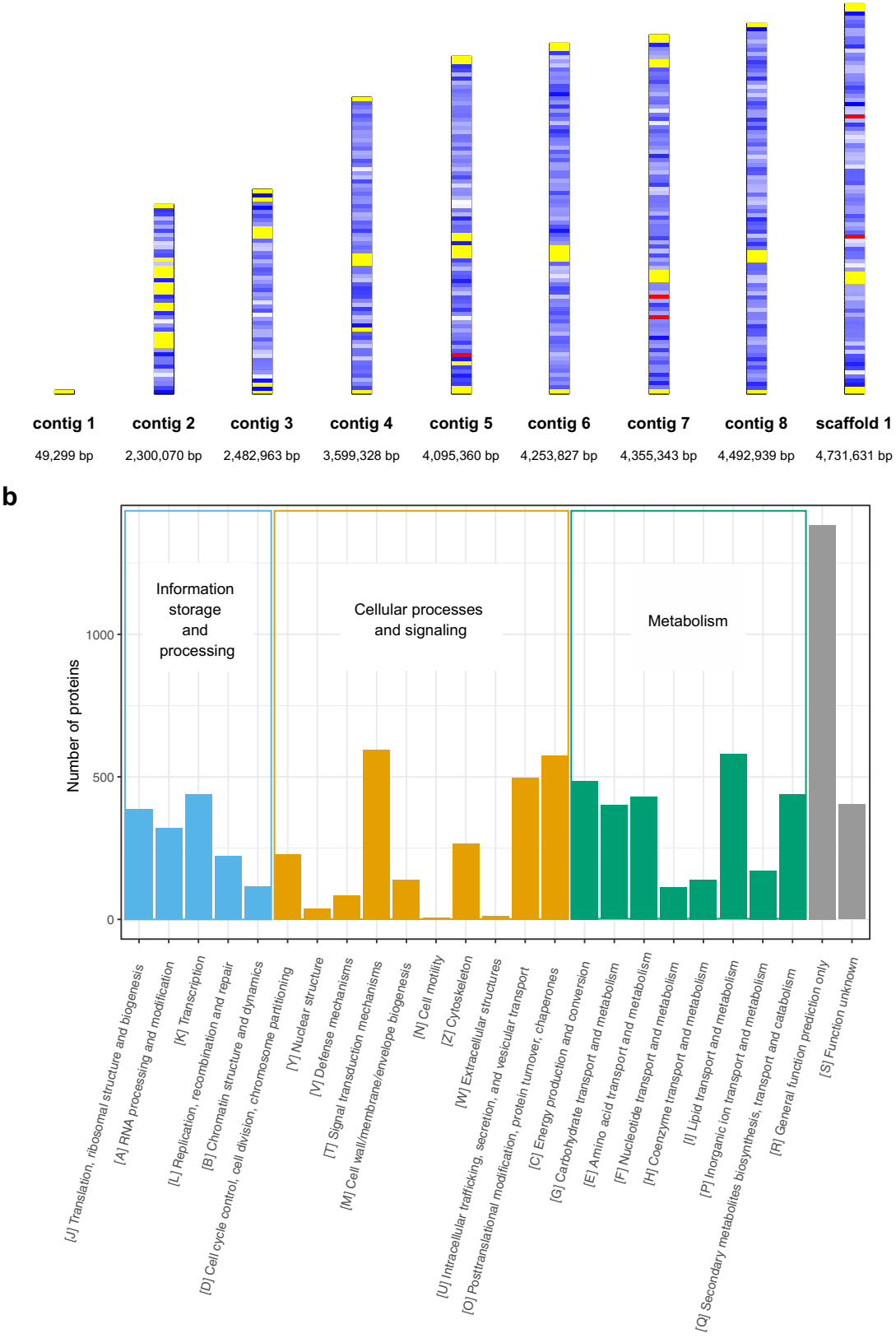

throughout the analysis (Table S3 of Online Resource 1). The example of such a sequence is the gene of a putative hexokinase (gene ID: HFD88_003314), for which the best BLASTP hits in the non-redundant protein database were the industrial progenitor strain Penicillium chrysogenum P2niaD18 and Penicillium rubens 43M1 (80\% identity in both cases), whereas no A. terreus counterparts could be found. Another example is the gene of a putative catalase (gene ID: HFD88_003313), which closely resembles the sequences found in other Aspergillus species (e.g., $89 \%$ identity with Aspergillus ruber CBS 135680 gene) but was not detected in any available genome of $A$. terreus. Interestingly, the genome of A. terreus ATCC 20542 carries a putative amidase gene that is generally missing in Aspergilli but exhibits more than $55 \%$ identity with the sequences of more evolutionarily distant fungal genera, namely Exophiala, Fonsecaea, and Cladophialophora.

\section{Secondary metabolism}

On the basis of the prediction, A. terreus ATCC 20542 genome encodes 74 secondary metabolite biosynthesis gene clusters (also referred to as "regions" according to the nomenclature used in antiSMASH 5 genome mining pipeline). Each of these regions is represented by at least one core biosynthetic gene representing polyketide synthase (PKS), non-ribosomal 
Table 2 Characteristics of complete A. terreus genomes deposited at the NCBI database. Genes were predicted by Augustus

\begin{tabular}{lllllll}
\hline Strain & GenBank assembly accession & $\begin{array}{l}\text { Total genome } \\
\text { size (bp) }\end{array}$ & $\begin{array}{l}\text { Number of contigs/ } \\
\text { scaffolds }\end{array}$ & N50 (bp) & $\begin{array}{l}\text { \%GC } \\
\text { Number of predicted } \\
\text { protein-coding genes }\end{array}$ \\
\hline NIH 2624 & GCA_000149615.1 & $29,364,022$ & 27 & $1,912,493$ & 52.6 & 10,413 \\
45A & GCA_001630395.1 & $29,325,441$ & 26 & $1,912,382$ & 51.1 & 10,219 \\
w25 & GCA_002749855.1 & $29,423,947$ & 536 & 326,294 & 52.7 & 10,436 \\
T3 Kankrej & GCA_002930435.1 & $27,963,153$ & 13,340 & 3497 & 52.1 & 13,346 \\
TN-484 & GCA_009014675.1 & $29,615,077$ & 1211 & 673,704 & 52.4 & 10,347 \\
M6925 & GCA_009834425.1 & $31,839,144$ & 35 & $4,133,337$ & 52.2 & 10,798 \\
IFO 6365 & GCA_009932835.1 & $28,667,710$ & 23 & $2,163,102$ & 53.0 & 10,227 \\
\hline
\end{tabular}

peptide synthetase (NRPS), terpene cyclase (TC), dimethylallyl tryptophan synthase (DMATS), or hybrids of various biosynthetic groups. These clusters are encoded on every A. terreus ATCC 20542 contig and one scaffold, except for the smallest, contig 1 (the Online Resource 1: Fig. S1). When other A. terreus genomes are considered, it can be noticed that the total number of predicted core biosynthetic genes involved in secondary metabolite (SM) production varied from 94 in A. terreus IFO 6365 to 99 in A. terreus M6925 (Table 4). Analysis of conservation of the core biosynthetic genes has shown that 78 of them are present in all of the analyzed $A$. terreus genomes (Fig. 3a). The genome of the main subject of this study, namely A. terreus ATCC 20542, is predicted to encode four unique core genes. The highest number (10) of unique sequences is encoded by the A. terreus IFO 6365 and TN-484 genomes (Fig. 3 a and b). Moreover, these two genomes do not encode a large group of over $15 \mathrm{SM}$ core genes which are conserved in other A. terreus genomes (Fig. 3b).

Analysis of conservation pattern of core SM synthases, which were experimentally linked to downstream products (as reviewed by Romsdahl and Wang 2019), has highlighted some distinctive features among the A. terreus genomes (Fig. 4a). For example, A. terreus ATCC 20542 genome does not encode the terretonin gene cluster (the core gene and the sequences of accessory proteins are all missing). The A. terreus IFO 6365 and TN-484 genomes, which are also the most phylogenetically distant from the other A. terreus genomes, do not encode acetylaranotin (ataP), (+)-geodin $($ ged $C)$, phenguignardic acid (pgnA), lovastatin $(\operatorname{lov} B$ and $\operatorname{lov} F)$, nor the asterrelenin, epi-aszonalenin A (anaPS) genes. The core genes of acetylaranotin production turned out to be the least conserved among the sequences considered in the present analysis, as they are missing in three out of seven investigated
Fig. 2 Phylogenetic tree of A. terreus strains based on ANI analysis performed using PYANI (0.2.9) python program employing BLAST+ program (Camacho et al. 2009; Pritchard et al. 2016). The UPGM tree based on ANI-1 values was calculated using phangorn R. package (Schliep 2011). The A. terreus ATCC 64974 was used as an outgroup. The tree was drawn in FigTree program (v. 1.4.4). The scale bar represents the sequence divergence

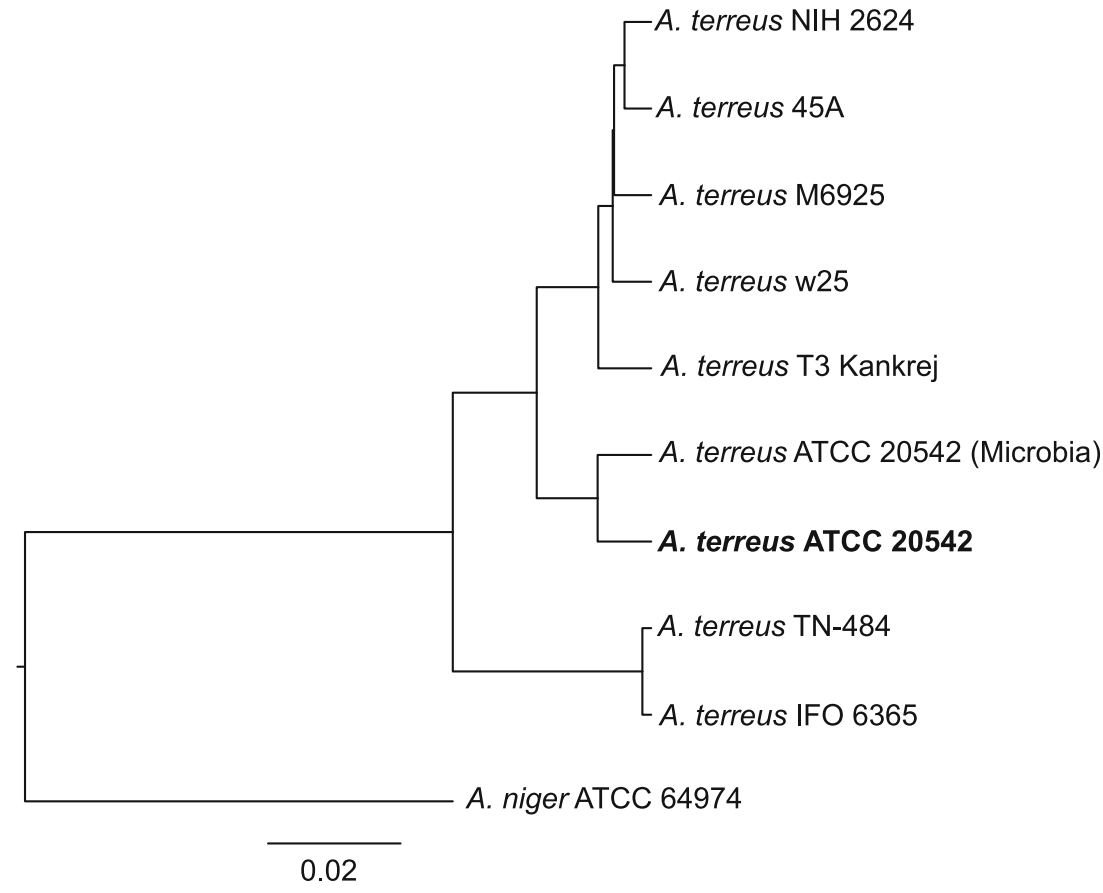


Table 3 Number of carbohydrate active enzymes predicted in A. terreus genomes. Assignment of the CAZy families to polysaccharides was performed according to de Vries et al. (2017)

\begin{tabular}{|c|c|c|c|c|c|c|c|c|}
\hline & Cellulose & Xylan & Galactomannan & Xyloglucan & Pectin & Starch & Inulin & Total \\
\hline $\begin{array}{l}\text { CAZy } \\
\text { families/St- } \\
\text { rain }\end{array}$ & $\begin{array}{l}\mathrm{GH} 5 \_4, \mathrm{GH} 5 \_5, \\
\text { GH5_22, GH6, } \\
\text { GH7, GH45, } \\
\text { AA9 }\end{array}$ & $\begin{array}{l}\text { GH10, GH11, } \\
\text { GH62, GH67, } \\
\text { GH115, CE1, } \\
\text { CE15 }\end{array}$ & $\begin{array}{l}\text { GH5_7, GH26, } \\
\text { GH27, } \\
\text { GH36, } \\
\text { GH134 }\end{array}$ & $\begin{array}{l}\text { GH12, } \\
\text { GH29, } \\
\text { GH74, } \\
\text { GH95 }\end{array}$ & $\begin{array}{l}\text { GH28, GH53, GH78, GH88, } \\
\text { GH93, GH105, PL1, PL3, } \\
\text { PL4, PL9, PL11, PL22, CE8, } \\
\text { CE12 }\end{array}$ & $\begin{array}{l}\text { GH13_1, GH13_5, } \\
\text { GH13_40, } \\
\text { GH15, GH31, } \\
\text { GH133 }\end{array}$ & GH32 & All \\
\hline ATCC 20542 & 23 & 18 & 11 & 9 & 36 & 23 & 6 & 466 \\
\hline NIH 2624 & 23 & 18 & 12 & 9 & 35 & 22 & 6 & 467 \\
\hline $45 \mathrm{~A}$ & 23 & 18 & 11 & 9 & 34 & 22 & 6 & 463 \\
\hline w25 & 23 & 18 & 11 & 10 & 37 & 22 & 6 & 474 \\
\hline IFO 6365 & 22 & 18 & 11 & 9 & 33 & 21 & 6 & 450 \\
\hline TN-484 & 22 & 18 & 11 & 9 & 33 & 21 & 6 & 452 \\
\hline M6925 & 23 & 17 & 11 & 10 & 37 & 23 & 11 & 483 \\
\hline
\end{tabular}

A. terreus strains (Fig. 4a). The biosynthetic gene cluster of lovastatin, the key secondary metabolite produced by A. terreus, was demonstrated here to be well-conserved among the five of the sequenced genomes of this species (Fig. 4b). Still, several single-nucleotide polymorphisms (SNPs) could be noticed along the cluster (Fig. 4b). The genes lovI and $l o v F$ turned out to be merged into a single sequence in A. terreus $45 \mathrm{~A}$ genome, but this may be due to incorrect in silico predictions and still requires further experimental verification.

Since the genome of A. terreus ATCC 20542 is the main focus of this study, a putative biosynthetic gene cluster that, as a whole, is unique to the newly sequenced strain was further analyzed in detail. This cluster encompasses the sequences spanning from HFD88_004290 to HFD88_004297 (contig 4th: 885,353..929,704) with HFD88_004294 (annotated as a PKS sequence) as the core biosynthetic gene. The amino acid sequence of the putative PKS encoded by HFD88_004294 has counterparts neither in the sequenced $A$. terreus strains, nor in any genome previously deposited into the NCBI. In this case, the closest BLASTP hit against the non-redundant protein database is the sequence found in Aspergillus brasiliensis (66\% identity at $68 \%$ query coverage). For comparison, if the sequence of lovastatin nonaketide synthase (LovB) originating from A. terreus NIH2624 is used as a query in BLASTP search using the same database, the percentage identity with the LovB counterpart found previously in A. terreus ATCC 20542 (Hendrickson et al. 1999) reaches as much as $98 \%$ at $100 \%$ query coverage. As far as the remaining regions of the above-mentioned unique cluster are concerned, three sequences in this region (HFD88_004290, HFD88_004291 and HFD88_004293) display close resemblance (more than $90 \%$ identity) with the proteins encoded in the genome of Aspergillus caelatus, whereas for HFD88_004295 and HFD88_004297, the best BLASTP hits were recorded in the genome of Coccidioides posadasii. For HFD88_004296, the most similar protein was detected in A. brasiliensis, similarly as for the core biosynthetic protein. Finally, there is only one sequence in this region that has its well-conserved counterpart in a strain representing the $A$. terreus species, namely HFD88_004292, which is 98\% identical with the corresponding orthologous protein in A. terreus NIH 2624.

Table 4 Core biosynthetic genes of A. terreus strains predicted by antiSMASH based on Augustus annotations

\begin{tabular}{lllllllll}
\hline Type/strain & $\begin{array}{l}\text { Type I iterative } \\
\text { PKS }\end{array}$ & NRPS & $\begin{array}{l}\text { NRPS- } \\
\text { like }\end{array}$ & $\begin{array}{l}\text { PKS/NRPS- } \\
\text { like }\end{array}$ & Hybrid & $\begin{array}{l}\text { TC } \\
\text { (terpene) }\end{array}$ & $\begin{array}{l}\text { DMATS } \\
\text { (indole) }\end{array}$ & $\begin{array}{l}\text { Total number core } \\
\text { biosynthetic genes }\end{array}$ \\
\hline ATCC 20542 & 25 & 24 & 22 & 5 & 2 & 8 & 10 & 96 \\
NIH 2624 & 27 & 19 & 26 & 3 & 2 & 10 & 10 & 97 \\
45A & 26 & 19 & 25 & 4 & 2 & 9 & 10 & 95 \\
w25 & 28 & 19 & 25 & 3 & 2 & 10 & 10 & 97 \\
IFO 6365 & 27 & 15 & 29 & 2 & 5 & 8 & 9 & 94 \\
TN-484 & 28 & 16 & 29 & 2 & 5 & 8 & 10 & 98 \\
M6925 & 26 & 20 & 29 & 3 & 2 & 9 & 99 \\
\hline
\end{tabular}

PKS polyketide synthase, NRPS non-ribosomal peptide synthetase, $T C$ terpene cyclase, DMATS dimethylallyl tryptophan synthase 
Fig. 3 Comparison of the predicted secondary metabolism potential in A. terreus strains. a Venn diagram of SM proteins from 5 representative genomes of each clade. The representative genomes were selected based on the phylogenetic analysis and genome quality. b Clustering of the A. terreus strains based on presence (blue tiles)/absence (gray tiles) of orthologous core biosynthetic proteins involved in secondary metabolism predicted by antiSMASH in every genome. Dendrogram was generated based on hierarchical clustering analysis. $Y$-axis: strain clustering; $x$-axis: protein clustering (dendrogram and orthologous groups not shown). A. terreus T3 Kankrej was not included in the analysis due to unsatisfactory quality of its genome (high sequence fragmentation) a

A. terreus IFO 6365

A. terreus ATCC 20542

b

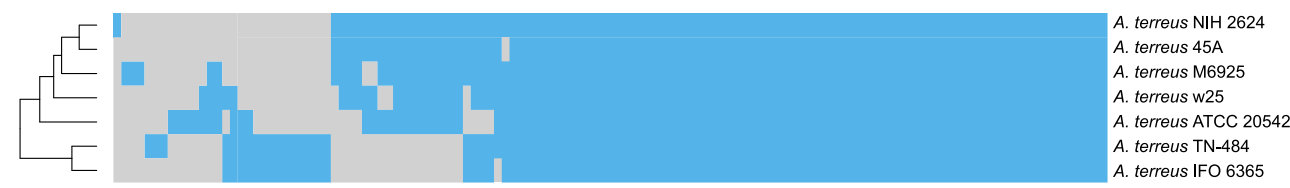

A. terreus $\mathrm{NIH} 2624$ a

b

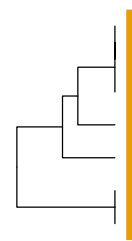

A. terreus $\mathrm{NIH} 2624$

A. terreus $45 \mathrm{~A}$

A. terreus $\mathrm{M} 6925$

A. terreus ATCC 20542

A. terreus w25

A. terreus $\mathrm{TN}-484$

A. terreus IFO 6365

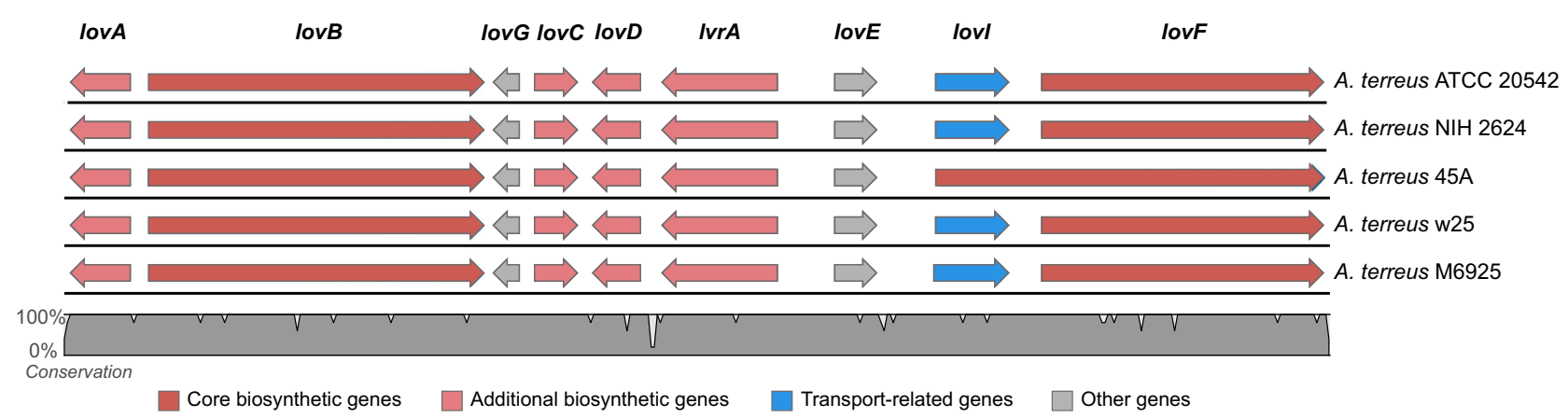

Fig. 4 Comparison of the characterized secondary metabolism gene clusters in A. terreus strains. a Clustering of the A. terreus strains based on presence (blue tiles)/absence (gray tiles) of orthologs of A. terreus NIH 2624 core biosynthetic proteins involved in secondary metabolism, which were experimentally linked to their metabolic products in previous studies (as reviewed by Romsdahl and Wang 2019). Dendrogram was generated based on hierarchical clustering analysis. $Y$-axis: strain clustering; $x$-axis: protein clustering (dendrogram not shown). A. terreus T3 Kankrej was not included in the analysis due to unsatisfactory quality of its genome (high sequence fragmentation). b Comparison of lovastatin biosynthesis gene cluster conserved in five $A$. terreus genomes (gene names according to Dietrich and Vederas 2014) 


\section{Discussion}

Until now, only a small portion (138,520 bp) of the A. terreus ATCC 20542 genome was made available to the public (provided by Microbia company). To fill this information gap and to provide the scientific community with the genome of this industrially important strain, we sequenced it using a hybrid approach consisting of long (Oxford Nanopore Technologies (ONT), Oxford, UK) and short read sequences (Illumina, San Diego, USA). The resulting assembly was of $30.4 \mathrm{Mb}$ and consisted of 8 contigs and 1 scaffold. Comparison with other A. terreus assemblies has shown that, in terms of the continuity-related quality of the genome, there are no previously released $A$. terreus assemblies that could be regarded as superior to the one presented in the current work (Table 2). Moreover, the analysis revealed that the size and general features of the A. terreus ATCC 20542 genome are comparable with other sequenced $A$. terreus genomes.

Availability of the whole genome sequence of the A. terreus strains opened the possibility to precisely measure the level of similarity displayed within this species. Phylogenetic analysis of the eight $A$. terreus strains highlighted the considerable genomic distance between them. The phylogenetic tree built on the ANI values consisted of three main clades, where the most occupied clade was represented by the A. terreus NIH 2624 strain and 4 other strains. On this tree, A. terreus ATCC 20542 formed a separate clade, and the most distant clade from the two clades was represented by the A. terreus IFO 6365 and A. terreus TN-484 strains. These findings suggested that the genetic differences between the A. terreus strains may be greater than expected and that they may directly influence the biosynthetic capabilities of these strains, which make them worth exploring.

First, we focused on the primary metabolism. According to the annotation presented in the current study, A. terreus ATCC 20542 is equipped with genes of catabolic enzymes allowing for the utilization of a broad set of carbon sources such as lactose and D-galactose (Table S1 of Online Resource 1). Among these carbon sources, it should be pointed out that lactose is the substrate of exceptional importance with regard to A. terreus ATCC 20542 cultivations. This slowly metabolized disaccharide was considered by many authors to be the recommended carbon source in the context of elevating lovastatin titers (Mulder et al. 2015). The catabolism of lactose encompasses several enzymatic steps, starting with its hydrolysis catalyzed by $\beta$-galactosidase to yield D-glucose (which then enters glycolysis) and D-galactose. In most eukaryotic organisms, D-galactose undergoes conversion into Dglucose-1-phosphate by the catalytic action of galactokinase, galactose-1-phosphate uridylyltransferase, and UDPgalactose 4-epimerase (Seiboth et al. 2007). The genes encoding the enzymes responsible for this metabolic route, known as the Leloir pathway, have all been found in the annotated genome of A. terreus ATCC 20542 (Table S1 of Online Resource 1). It is worth mentioning that these three genes were detected only in single copies, what agrees well with the findings of de Vries et al. (2007), who previously examined 19 genomes of various species representing the Aspergillus genus. In the mentioned study, the sequences of galactokinase and UDP-galactose 4-epimerase were found in a single copy in all the investigated assemblies, whereas the gene of galactose-1-phosphate uridylyltransferase turned out to be present in two copies in Aspergillus glaucus and in a single copy in the remaining 18 Aspergilli.

In a species-wide perspective, the comparative analysis revealed only a modest degree of diversity with respect to the presence and the number of genes involved in carbon source utilization in A. terreus strains (Table S1 of Online Resource 1). In some cases, the number of orthologous sequences associated with a given enzymatic function was not identical in all the investigated fungi (e.g., the number of invertase genes varied from 1 to 2 depending on the microorganism), but for most sequences, encoding the sugar catabolic enzymes, no diversity was recorded in this respect (Table S1 of Online Resource 1). Similar observations were made with regard to the genes involved in the production of organic acids, where even slight differences in gene copy numbers were very rare among the tested strains (Table S2 of Online Resource 1). Moreover, evaluation of the plant biomass-degrading ability across A. terreus strains, based on presence of the genes from CAZy families, has shown some modest degree of diversity among them. Especially the genome of A. terreus M6925 was found to harbor the highest number of CAZy genes among all of the investigated strains. Moreover, the two phylogenetically most distant strains, namely $A$. terreus IFO 6365 and TN484 , were predicted to have slightly poorer enzyme repertoire for the degradation of cellulose, pectin, and starch, in comparison with other A. terreus species. In general, these results may be seen as a correlation between the phylogenetic relationship between the strains and the similarity of genetic inventories related to plant biomass degradation.

All in all, as far as the catabolic enzyme-encoding genes associated with the metabolism of sugars, organic acids, and plant biomass components are concerned, this functionally related part of the A. terreus genome is rather well-conserved at the species level and the genetic diversity among the strains of $A$. terreus can be regarded as relatively low. However, some subtle, yet distinguishable features for each strain can always be found. For example, in the newly sequenced genome of A. terreus ATCC 20542, we found several unique genes coding for, e.g., hexokinase, catalase, or amidase which had no counterparts among $A$. terreus strains bet shared similarity with more evolutionarily distant fungal genera (Table S3 of Online Resource 1). One cannot exclude that these unique genes played a role in the survival of A. terreus ATCC 
20542 in its natural environment and contributed to its overall fitness and metabolic capabilities.

The next metabolic pathways which were analyzed were those related to secondary metabolism. Compared with other strains, the newly sequenced genome of A. terreus ATCC 20542 turned out to be particularly rich in NRPS genes, what might reflect the potential of this microorganisms to produce a relatively wide spectrum of non-ribosomal peptides (Table 4). According to the antiSMASH analysis performed in the present work, all of the investigated $A$. terreus strains are equipped with 10 DMATS genes except $A$. terreus IFO6365, which has only eight genes of this kind. As recently mentioned by Takahashi at el. (2020), A. terreus IFO6365 is "one of the highest-yielding itaconic acid-producing wild-type strains". It is interesting that the mutant derived from this strain, namely A. terreus TN-484, is more enriched with the core biosynthetic genes associated with SM production than its parental strain, having more genes encoding PKS, NRPS, and TC enzymes (Table 4). In fact, the genomes of $A$. terreus TN-484 and $A$. terreus w25 harbor the largest number of PKS genes among all the investigated datasets (Table 4). Having a broader set of biosynthetic genes could be seen as an unnecessary burden for a mutant strain tailored specifically towards the efficient production of itaconic acid, even if these extra genes remained silent under typical cultivation conditions. When conservation pattern of the core biosynthetic genes was analyzed, it was revealed that it followed the phylogenetic relationship among the A. terreus strains (Fig. 3b). Considering the number of core genes conserved only among a limited number of strains or present solely in a single strain, one may expect a strain-specific catalog of secondary metabolites exhibited by the representatives of this fungal species. Particularly, the A. terreus IFO 6365 and TN-484 genomes displayed here the most distinguishable features. These genomes were not only found to harbor the highest number of unique sequences but also the largest group of core SM genes was not conserved in them. Among the absent genes encoding some of the experimentally verified SM clusters, such as those of lovastatin and (+)-geodin (Fig. 4a). Since lovastatin and $(+)$-geodin are the secondary metabolites produced in relatively large quantities in A. terreus cultures (Bizukojc and Ledakowicz 2007; Hasan et al. 2019), the lack of genes responsible for the biosynthesis of these two molecules in itaconic acid producers A. terreus IFO 6365 and TN-484 can be viewed as a relief of the unnecessary biosynthetic load in favor of maximizing itaconic acid yields. Several features related to SM synthesis were also discovered in the A. terreus ATCC 20542 genome. For example, it lacks terretonin gene cluster but harbors four unique core genes. By further investigating of these genes, a completely unique gene cluster was exposed, which as a whole is present only in A. terreus ATCC 20542 and no other A. terreus strains. The core biosynthetic gene of this cluster, a putative PKS sequence, was found to be particularly unique since it has no counterparts in any of the genomes deposited at the NCBI database. This cluster can be regarded as interesting targets of future experimental efforts towards uncovering novel secondary metabolites of potential industrial and pharmaceutical interest.

The predicted absence of the core genes from the terretonin and (+)-geodin pathway in A. terreus ATCC 20542 and both, A. terreus IFO 6365 and TN-484, respectively, may be important in the context of invasive fungal infections, since these metabolites have been identified as pathogenicity factors in A. terreus (reviewed in Bengyella et al. 2017 and Shankar et al. 2018). It is possible that the absence of these genes as well as those of other predicted SM gene clusters may influence pathogenic potential of these strains. Indeed, A. terreus ATCC 20542 is classified as unlikely to cause disease (Biosafety level 1, BSL1). Further research, however, is needed to verify and to relate the absence of these metabolites with pathogenicity of these strains. Nevertheless, the SM gene clusters predicted in this study may constitute a valuable resource for searching of new pathogenic factors which would distinguish pathogenic strains from non-pathogenic ones, since the key virulent proteins which were identified in a proteomic study of A. terreus (Thakur and Shankar 2017) are all present in the genomes of A. terreus strains (Table S4 of Online Resource 1). It is also possible that sequence polymorphism at the regulatory regions modulating expression of these genes may exist. In order to identify it and to investigate its influence, more studies comparing expression level of these genes among the $A$. terreus strains are needed.

As discussed above, the genome of A. terreus ATCC 20542 harbors several genes that can be regarded as unique among the sequenced genomes of $A$. terreus. Further investigation of the biological events associated with these sequences, e.g., the analysis of expression, metabolic profiles recorded for the deletion mutants or gene-by-gene elucidation of the biosynthetic gene clusters, would surely provide insights into the underlying molecular mechanisms associated with the phenotypic diversity at the species level. Furthermore, the complete genome of a model lovastatinproducing strain will open the door for the system-scale studies on lovastatin biosynthesis, possibly leading to improved, highly efficient fungal cell factories obtained via metabolic engineering. An interesting study would be to comprehensively compare the wild-type parental strain with its progeny of industrial mutants obtained mostly by random mutagenesis to gain insights into cellular changes that ultimately lead to increased lovastatin titers, productivities, and yields. Unfortunately, since the complete genomes of lovastatinproducing industrial mutants of $A$. terreus have not been released so far, the analysis of this kind is not feasible at the moment. Addressing the genomic diversity of A. terreus, there are considerable differences among the genetic inventories associated with secondary metabolic pathways in the strains 
belonging to this species. Moreover, there are certain links between the known characteristics of the investigated fungi and their genomic features, e.g. the lack of lovastatin and (+)-geodin clusters in the strains used for the production of itaconic acid. As the predictions regarding the biosynthetic gene clusters indicate, understanding the species-level biosynthetic diversity in $A$. terreus can be expected to facilitate the process of strain identification based on metabolic profiles.

To conclude, the present study provides the complete genome sequence of lovastatin-producing wild-type strain A. terreus ATCC 20542. In terms of continuity, this is the dataset of highest quality among the released A. terreus genomes. The assembled and annotated genome of this model microorganism will serve as a future reference for studying the biosynthesis of lovastatin and other fungal secondary metabolites. The comparative study conducted here shows that the diversity among A. terreus strains is more evident in the genomic areas associated with secondary metabolism than in the segments encoding the genes related to primary metabolic pathways; however, the differences in the number of highly conserved catabolic genes are also clearly visible. Finally, the newly sequenced genome of A. terreus ATCC 20542 harbors several unique sequences that are interesting subjects for future research on fungal metabolism, especially in the comparative context.

Supplementary Information The online version contains supplementary material available at https://doi.org/10.1007/s00253-021-11133-0.

Acknowledgements We are grateful to Institute of Biochemistry and Biophysics, Polish Academy of Sciences, Warsaw, Poland, for sequencing and assembling of the A. terreus ATCC 20542 genome. This study was funded by the National Science Centre (Poland) (grant number 2017/27/B/NZ9/00534).

Author contribution TB and MB conceived and designed the study. TB conducted strain cultivation. MR performed genome annotation and comparative analysis. TB and MR wrote the original draft. MB reviewed the draft. All authors read and approved the manuscript.

Funding This study was funded by the National Science Centre (Poland) (grant number 2017/27/B/NZ9/00534).

Data availability The sequence of $A$. terreus ATCC 20542 genome is accessible through NCBI database under the BioProject: PRJNA622971.

\section{Compliance with ethical standards}

Ethics approval and consent to participate This article does not contain any studies with human participants or animals performed by any of the authors. Consent to participate is not applicable.

\section{Consent for publication Not applicable.}

Conflict of interest The authors declare that they have no conflict of interest.
Open Access This article is licensed under a Creative Commons Attribution 4.0 International License, which permits use, sharing, adaptation, distribution and reproduction in any medium or format, as long as you give appropriate credit to the original author(s) and the source, provide a link to the Creative Commons licence, and indicate if changes were made. The images or other third party material in this article are included in the article's Creative Commons licence, unless indicated otherwise in a credit line to the material. If material is not included in the article's Creative Commons licence and your intended use is not permitted by statutory regulation or exceeds the permitted use, you will need to obtain permission directly from the copyright holder. To view a copy of this licence, visit http://creativecommons.org/licenses/by/4.0/.

\section{References}

Alberts AW, Chen J, Kuron G, Hunt V, Huff J, Hoffman C, Rothrock J, Lopez M, Joshua H, Harris E, Patchett A, Monaghan R, Currie S, Stapley E, Albers-Schonberg G, Hensens O, Hirshfield J, Hoogsteen K, Liesch J, Springer J (1980) Mevinolin: a highly potent competitive inhibitor of hydroxymethylglutaryl-coenzyme A reductase and a cholesterol-lowering agent. Proc Natl Acad Sci U S A 77:39573961

Almagro Armenteros JJ, Tsirigos KD, Sønderby CK, Petersen TN, Winther O, Brunak S, von Heijne G, Nielsen H (2019) SignalP 5.0 improves signal peptide predictions using deep neural networks. Nat Biotechnol 37:420-423

Andrews S (2010) FastQC: a quality control tool for high throughput sequence data. Available online at: http://www.bioinformatics. babraham.ac.uk/projects/fastqc

Askenazi M, Driggers EM, Holtzman DA, Norman TC, Iverson S, Zimmer DP, Boers M-E, Blomquist PR, Martinez EJ, Monreal AW, Feibelman TP, Mayorga ME, Maxon ME, Sykes K, Tobin JV, Cordero E, Salama SR, Trueheart J, Royer JC, Madden KT (2003) Integrating transcriptional and metabolite profiles to direct the engineering of lovastatin-producing fungal strains. Nat Biotechnol 21:150-156

Barrios-González J, Miranda RU (2010) Biotechnological production and applications of statins. Appl Microbiol Biotechnol 85:869-883

Bengyella L, Yekwa EL, Subhani MN, Tambo E, Nawaz K, Hetsa BA, Iftikhar S, Walkhom SD, Roy P (2017) Invasive Aspergillus terreus morphological transitions and immunoadaptations mediating antifungal resistance. Infect Drug Resist 10:425-436

Bizukojc M, Ledakowicz S (2007) Simultaneous biosynthesis of (+)geodin by a lovastatin-producing fungus Aspergillus terreus. J Biotechnol 132:453-460

Blin K, Shaw S, Steinke K, Villebro R, Ziemert N, Lee SY, Medema MH, Weber T (2019) AntiSMASH 5.0: updates to the secondary metabolite genome mining pipeline. Nucleic Acids Res 47:W81-W87

Boruta T, Bizukojc M (2014) Culture-based and sequence-based insights into biosynthesis of secondary metabolites by Aspergillus terreus ATCC 20542. J Biotechnol 175:53-62

Boruta T, Bizukojc M (2016) Induction of secondary metabolism of Aspergillus terreus ATCC 20542 in the batch bioreactor cultures. Appl Microbiol Biotechnol 100:3009-3022

Buckland B, Gbewonyo K, Hallada T, Kaplan L, Masurekar P (1989) Production of lovastatin, an inhibitor of cholesterol accumulation in humans. In: Demain AL, Somkuti GA, Hunter-Cevera JC, Rossmore HW (eds) Novel Microbial Products for Medicine and Agriculture. Elsevier, New York, pp 161-169

Camacho C, Coulouris G, Avagyan V, Ma N, Papadopoulos J, Bealer K, Madden TL (2009) BLAST+: architecture and applications. BMC Bioinf 10:421

Chan PP, Lowe TM (2019) tRNAscan-SE: Searching for tRNA genes in genomic sequences. Methods Mol Biol 1962:1-14 
De Coster W, D'Hert S, Schultz DT, Cruts M, Van Broeckhoven C (2018) NanoPack: visualizing and processing long-read sequencing data. Bioinformatics 34:2666-2669

de Vries RP, Riley R, Wiebenga A, Aguilar-Osorio G, Amillis S, Uchima CA, Anderluh G, Asadollahi M, Askin M, Barry K, Battaglia E, Bayram Ö, Benocci T, Braus-Stromeyer SA, Caldana C, Cánovas D, Cerqueira GC, Chen F, Chen W, Choi C, Clum A, dos Santos RAC, de Lima Damásio AR, Diallinas G, Emri T, Fekete E, Flipphi M, Freyberg S, Gallo A, Gournas C, Habgood R, Hainaut M, Harispe ML, Henrissat B, Hildén KS, Hope R, Hossain A, Karabika E, Karaffa L, Karányi Z, Kraševec N, Kuo A, Kusch H, LaButti K, Lagendijk EL, Lapidus A, Levasseur A, Lindquist E, Lipzen A, Logrieco AF, MacCabe A, Mäkelä MR, Malavazi I, Melin P, Meyer V, Mielnichuk N, Miskei M, Molnár ÁP, Mulé G, Ngan CY, Orejas M, Orosz E, Ouedraogo JP, Overkamp KM, Park HS, Perrone G, Piumi F, Punt PJ, Ram AFJ, Ramón A, Rauscher S, Record E, Riaño-Pachón DM, Robert V, Röhrig J, Ruller R, Salamov A, Salih NS, Samson RA, Sándor E, Sanguinetti M, Schütze T, Sepčić K, Shelest E, Sherlock G, Sophianopoulou V, Squina FM, Sun H, Susca A, Todd RB, Tsang A, Unkles SE, van de Wiele N, van Rossen-Uffink D, de Castro Oliveira JV, Vesth TC, Visser J, Yu JH, Zhou M, Andersen MR, Archer DB, Baker SE, Benoit I, Brakhage AA, Braus GH, Fischer R, Frisvad JC, Goldman GH, Houbraken J, Oakley B, Pócsi I, Scazzocchio C, Seiboth B, vanKuyk PA, Wortman J, Dyer PS, Grigoriev IV (2017) Comparative genomics reveals high biological diversity and specific adaptations in the industrially and medically important fungal genus Aspergillus. Genome Biol 18(1):28

Dietrich D, Vederas JC (2014) Lovastatin, compactin, and related anticholesterolemic agents. In: Martín JF, García-Estrada C, Zeilinger S (eds) Biosynthesis and molecular genetics of fungal secondary metabolites. Fungal biology. Springer, New York

Endo A (2010) A historical perspective on the discovery of statins. Proc Jpn Acad Ser B 86:484-493

Ghanem NB, Yusef HH, Mahrouse HK (2000) Production of Aspergillus terreus xylanase in solid-state cultures: application of the PlackettBurman experimental design to evaluate nutritional requirement. Bioresour Technol 73:113-121

Hasan H, Abd Rahim MH, Campbell L, Carter D, Abbas A, Montoya A (2019) Improved lovastatin production by inhibiting (+)-geodin biosynthesis in Aspergillus terreus. New Biotechnol 52:19-24

Hendrickson L, Davis CR, Roach C, Nguyen DK, Aldrich T, McAda PC, Reeves CD (1999) Lovastatin biosynthesis in Aspergillus terreus: characterization of blocked mutants, enzyme activities and a multifunctional polyketide synthase gene. Chem Biol 6:429-439

Jones P, Binns D, Chang H-Y, Fraser M, Li W, McAnulla C, McWilliam H, Maslen J, Mitchell A, Nuka G et al (2014) InterProScan 5: genome-scale protein function classification. Bioinformatics 30 : $1236-1240$

Käll L, Krogh A, Sonnhammer ELL (2007) Advantages of combined transmembrane topology and signal peptide prediction-the Phobius web server. Nucleic Acids Res (Web Server issue):W429-W432

Kanamasa S, Minami T, Okabe M, Park EY, Fujimoto T, Takahashi A, Murase M, Fukuyoshi S, Oda A, Satou K, Takahashi H (2019) Draft genome sequence of Aspergillus terreus high-itaconic-acidproductivity mutant TN-484. Microbiol Resour Announc 8: e01170-e01119

Kennedy J, Auclair K, Kendrew SG, Park C, Vederas JC, Hutchinson CR (1999) Modulation of polyketide synthase activity by accessory proteins during lovastatin biosynthesis. Science 284:1368-1372

Kitagawa M, Higashi H, Takahashi IS, Okabe T, Ogino H, Taya Y, Hishimura S, Okuyama A (1994) A cyclin-dependent kinase inhibitor, butyrolactone I, inhibits phosphorylation of RB protein and cell cycle progression. Oncogene 9:2549-2557

Klement T, Buchs J (2013) Itaconic acid-a biotechnological process in change. Bioresour Technol 135:422-431
Kolmogorov M, Yuan J, Lin Y, Pevzner PA (2019) Assembly of long, error-prone reads using repeat graphs. Nat Biotechnol 37:540-546

Krull S, Hevekerl A, Kuenz A, Prüße U (2017) Process development of itaconic acid production by a natural wild type strain of Aspergillus terreus to reach industrially relevant final titers. Appl Microbiol Biotechnol 101:4063-4072

Lass-Flörl C (2018) Treatment of infections due to Aspergillus terreus species complex. J Fungi (Basel) 4(83)

Lechner M, Findeiss S, Steiner L, Marz M, Stadler PF, Prohaska SJ (2011) Proteinortho: detection of (co-)orthologs in large-scale analysis. BMC Bioinf 12:124

Lombard V, Golaconda Ramulu H, Drula E, Coutinho PM, Henrissat B (2014) The carbohydrate-active enzymes database (CAZy) in 2013. Nucleic Acids Res 42:D490-D495

Medema MH, Blin K, Cimermancic P, De Jager V, Zakrzewski P, Fischbach MA, Weber T, Takano E, Breitling R (2011) AntiSMASH: Rapid identification, annotation and analysis of secondary metabolite biosynthesis gene clusters in bacterial and fungal genome sequences. Nucleic Acids Res (Web Server issue):W339 W346

Mulder KCL, Mulinari F, Franco OL, Soares MSF, Magalhaes BS, Parachin NS (2015) Lovastatin production: from molecular basis to industrial process optimization. Biotechnol Adv 33:648-666

Nowak RM, Jastrzębski JP, Kuśmirek W, Sałamatin R, Rydzanicz M, Sobczyk-Kopcioł A, Sulima-Celińska A, Paukszto $Ł$, Makowczenko KG, Płoski R, Tkach VV, Basałaj K, Młocicki D (2019) Hybrid de novo whole-genome assembly and annotation of the model tapeworm Hymenolepis diminuta. Sci Data 6(1):302

Ohashi H, Akiyama H, Nishikori K, Mochizuki J (1992) Asterric acid, a new endothelin binding inhibitor. J Antibiot (Tokyo) 45:1684-1685

Okabe M, Lies D, Kanamasa S, Park EY (2009) Biotechnological production of itaconic acid and its biosynthesis in Aspergillus terreus. Appl Microbiol Biotechnol 84:597-606

Palanivel M, Mac Aogáin M, Purbojati RW, Uchida A, Aung NW, Lim SBY, Putra A, Drautz-Moses DI, Seaton S, Rogers TR, Schuster SC, Chotirmall SH (2020) Whole-genome sequencing of Aspergillus terreus species complex. Mycopathologia 185:405-408

Pastor FJ, Guarro J (2014) Treatment of Aspergillus terreus infections: a clinical problem not yet resolved. Int J Antimicrob Agents 44:281289

Pritchard L, Glover RH, Humphris S, Elphinstone JG, Toth IK (2016) Genomics and taxonomy in diagnostics for food security: softrotting enterobacterial plant pathogens. Anal Methods 8:12-24

Rinderknecht H, Ward JL, Bergel F, Morrison AL (1947) Studies on antibiotics: 2. Bacteriological activity and possible mode of action of certain non-nitrogenous natural and synthetic antibiotics. Biochem J 41:463-469

Romsdahl J, Wang CCC (2019) Recent advances in the genome mining of Aspergillus secondary metabolites (covering 2012-2018). MedChemComm 10:840-866

Sato S, Okusa N, Ogawa A, Ikenoue T, Seki T, Tsuji T (2005) Identification and preliminary SAR studies of (+)-geodin as a glucose uptake stimulator for rat adipocytes. J Antibiot 58:583-589

Savitha J, Bhargavi SD, Praveen VK (2016) Complete genome sequence of soil fungus Aspergillus terreus (KM017963), a potent lovastatin producer. Genome Announc 4:e00491-e00416

Schliep KP (2011) phangorn: phylogenetic analysis in R. Bioinformatics. 27(4):592-593

Seiboth B, Pakdaman BS, Hartl L, Kubicek CP (2007) Lactose metabolism in filamentous fungi: how to deal with an unknown substrate. Fungal Biol Rev 21:42-48

Sethi BK, Nanda PK, Sahoo S (2016) Characterization of biotechnologically relevant extracellular lipase produced by Aspergillus terreus NCFT 4269. 10. Braz J Microbiol 47:143-149

Shankar J, Tiwari S, Shishodia SK, Gangwar M, Hoda S, Thakur R, Vijayaraghavan P (2018) Molecular insights into development and 
virulence determinants of Aspergilli: a proteomic perspective. Front Cell Infect Microbiol 8:180

Shinohara C, Chikanishi T, Nakashima S, Hashimoto A, Hamanaka A, Endo A, Hasumi K (2000) Enhancement of fibrynolytic activity of vascular endothelial cells by chaetoglobosin $\mathrm{A}$, crinipellin $\mathrm{B}$, geodin and triticone B. J Antibiot 53:262-268

Sohail M, Ahmad A, Khan SA (2016) Production of cellulase from Aspergillus terreus MS105 on crude and commercially purified substrates. 3 Biotech 6:103

Stanke M, Waack S (2003) Gene prediction with a hidden Markov model and a new intron submodel. Bioinformatics 19:ii215-ii225

Steinbach WJ, Perfect JR, Schell WA, Walsh TJ, Benjamin DK Jr (2004) In vitro analyses, animal models, and 60 clinical cases of invasive Aspergillus terreus infection. Antimicrob Agents Chemother 48: 3217-3225

Takahashi H, Minami T, Okabe M, Park EY, Fujimoto T, Takahashi A, Murase M, Fukuyoshi S, Satou K, Kanamasa S (2020) Draft genome sequence of the Aspergillus terreus high-itaconic-acidproductivity strain IFO6365. Microbiol Resour Announc 9: e00080-e00020

Takatsuki A, Yamaguchi I, Tamura G, Misato T, Arima K (1969) Correlation between the anti-animal and anti-plant-virus activities of several antibiotics. (Studies on antiviral and antitumor antibiotics. XIX). J Antibiot (Tokyo) 22:442-445
Thakur R, Shankar J (2017) Proteome profile of Aspergillus terreus conidia at germinating stage: identification of probable virulent factors and enzymes from mycotoxin pathways. Mycopathologia 182:771784

Vinci VA, Hoerner TD, Coffman AD, Schimmel TG, Dabora RL, Kirpekar AC, Ruby CL, Stieber RW (1991) Mutants of a lovastatin-hyperproducing Aspergillus terreus deficient in the production of sulochrin. J Ind Microbiol 8:113-119

Vyas JM (2011) The duality of Aspergillus terreus: differential immune responses to distinct conidia. Virulence 2:181-184

Wilson K (1987) Preparation of genomic DNA from bacteria. In: Ausubel FM, Bent R, Kingston RE, Moore DD, Seidman JG, Smith JA, Struhl K (eds) Current protocols in molecular biology pp. J. Wiley \& Sons, New York, pp 2.4.1-2.4.5

Wu S, Zhu Z, Fu L, Niu B, Li W (2011) WebMGA: a customizable web server for fast metagenomic sequence analysis. BMC Genomics 12 : 444

Zhang H, Yohe T, Huang L, Entwistle S, Wu P, Yang Z, Busk PK, Xu Y, Yin Y (2018) DbCAN2: A meta server for automated carbohydrateactive enzyme annotation. Nucleic Acids Res 46(W1):W95-W101

Publisher's note Springer Nature remains neutral with regard to jurisdictional claims in published maps and institutional affiliations. 